\title{
MulTileVEl ADDRESS REORganizATION TYPE2 In Wireless Personal AREA Network
}

\author{
Debabrato Giri ${ }^{1}$ and Uttam Kumar Roy ${ }^{2}$ \\ ${ }^{1}$ Department of Information Technology, Jadavpur University, Kolkata, India \\ debabrato.girietcs.com \\ ${ }^{2}$ Department of Information Technology, Jadavpur University, Kolkata, India \\ u_royeit.jusl.ac.in
}

\begin{abstract}
The Standard for Wireless personal area network (WPAN) has been given by IEEE 802.15.4-2003 these networks are consists of Low rate, Low powered, Low memory devices. ZigBee Alliance has provided Network layer specification and Physical layer (PHY) and Medium access control (MAC) specification has been given by IEEE. In general the networks are of two kind Tree/Mess. In tree network no routing table is required for routing. After the great success in PAN this technique has also been tried to apply in business Network too. The main problem with this routing is that the maximum no of child (Router capable or end device) at any level and maximum network depth is fixed and this is done at the time of network formation. So the network can't grow beyond that max limit of breadth and width. We have addressed the network depth problem in our paper "Address Borrowing in Wireless Personal Area Network". Now in some other network configuration the maximum breadth of the network may be attained but the maximum depth of the network may not be attained (because of the asymmetric nature of the physical area) at that part and hence address lies unused. Here In this paper we have provided a unified address reorganizing scheme which can be easily applied to tree network so that the network can grow beyond the maximum no of child present at any level and overcome the address exhaustion problem by reorganizing address as per the requirement but without adding any extra overhead of having a routing table.
\end{abstract}

\section{KEYWORDS}

PAN; Mesh; Address Reorganizing; Routing; WPAN; Tree;

\section{INTRODUCTION}

In recent past there has been a steady rise in wireless networking. Wireless sensor/actuator networks ("sensornets") represents a new computing class consisting of large number of nodes which are often embedded in their operating environments distributed over wide geographical area often in remote and largely inaccessible regions. The node themselves ranges from tiny, resource-constrained devices called motes to PDA-class computing devices that are capable of sensing, computation, communication, and actuation. Sensornets allows us to instrument, observe, and respond to the physical world on scales of space and time previously impossible. Standards like IEEE 802.11[11] (Wi-Fi) and IEEE 802.15.1 [13] (12) have come into existence. IEEE 802.11 targets high data rate, mains powered, high cost and relatively long range applications. Bluetooth is one of the first standards designed for low range, low power devices. Because of its huge popularity now a day all most all the mobiles are coming with Bluetooth. But the main problem with this technology is the data can be transferred to single hop only. As a result more and more low-cost high-quality devices appear in the market; short-range low-rate wireless personal area networks are poised to take the world in a way observed never before which can transfer the data using multiple hops. 
Wireless personal area networks (WPANs) are used to convey information over relatively short distances. Unlike wireless local area networks (WLANs), connections effected via WPANs involve little or no infrastructure. This feature allows small, power-efficient, inexpensive solutions to be implemented for a wide range of devices.

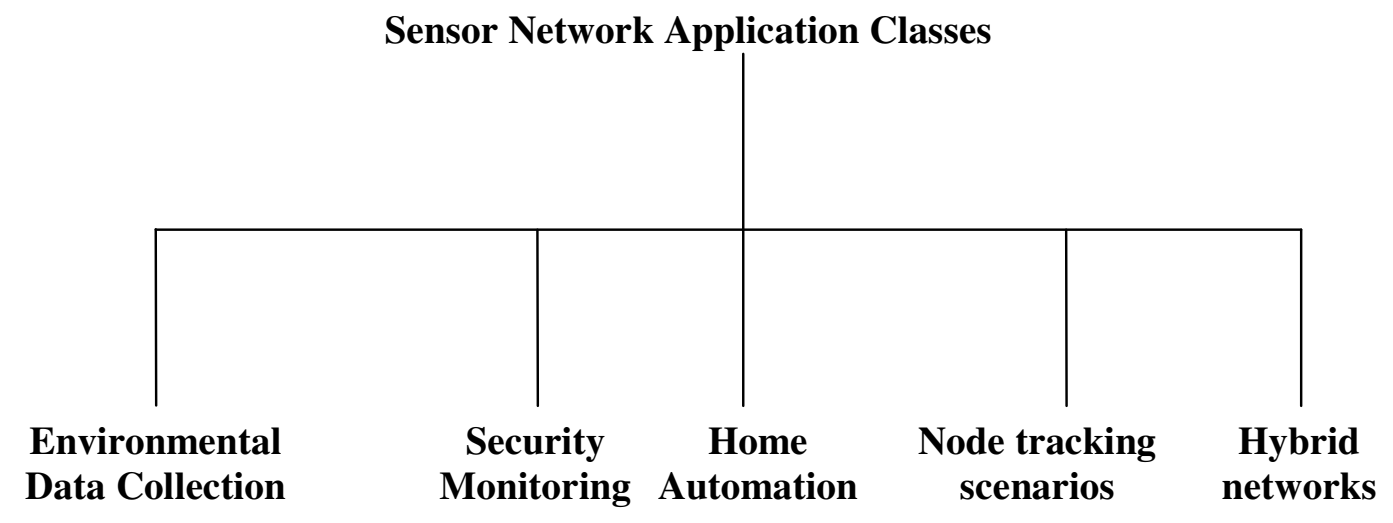

IEEE 802.15.4 [19] (henceforth referred to as 802.15.4) is a landmark in the attempt to bring ubiquitous networking [Figure 1] into our lives. Designed uniquely for energy-conscious low data rate appliances, it specifies the PHYsical (PHY) layer and MAC sub-layer of the protocol stack. The ZigBee Alliance [19] has defined the specification for the network (NWK), security and application profile layers for an 802.15.4-based system. Network layer supports three topologies Star, Tree and Mesh. The main advantage of tree address allocation is that it does not require any routing table to forward a message. In that simple mathematical equations are used for address assignment and routing.

With ZigBee devices on the horizon, ubiquitous networking looks elusive no more. It is not too distant a future one may chance to have one's home-appliances wedded together in a smart and cooperative network that allows them to talk to each other seamlessly. Sensors and actuators will communicate without barrier. They can be deployed pervasively in disaster-hit areas to monitor the situation to provide situational awareness and automatically take appropriate actions.

Some of its major application areas [Figure 1] are:

- Home automation

- Industrial control and monitoring

- Personal health-care

- Public safety including sensing, location determination and situational awareness at disaster sites

- Automotive sensing, such as tire pressure monitoring

- Precision agriculture such as the sensing of soil moisture, pesticide, herbicide, and $\mathrm{pH}$ levels.

- Mobile telecommunication such as peer-to-peer small data sharing, mobile commerce, mobile gaming, voice over ZigBee and chatting.

\section{A QUICK TOUR OF 802.15.4/ZIGBEE}

The following section gives a brief overview of ZigBee network formation and routing technique. 


\subsection{15.4/ZigBee target applications}

With ZigBee devices on the horizon, ubiquitous networking looks elusive no more. It is not too distant a future one may chance to have one's home-appliances wedded together in a smart and cooperative network that allows them to talk to each other seamlessly. Sensors and actuators will communicate without barrier. They can be deployed pervasively in disaster-hit areas to monitor the situation to provide situational awareness and automatically take appropriate actions.

Some of its major application areas [Figure 1] are:

- Home automation

- Industrial control and monitoring

- Personal health-care

- Public safety including sensing, location determination and situational awareness at disaster sites

- Automotive sensing, such as tire pressure monitoring

- Precision agriculture such as the sensing of soil moisture, pesticide, herbicide, and $\mathrm{pH}$ levels.

- Mobile telecommunication such as peer-to-peer small data sharing, mobile commerce, mobile gaming, voice over ZigBee and chatting.

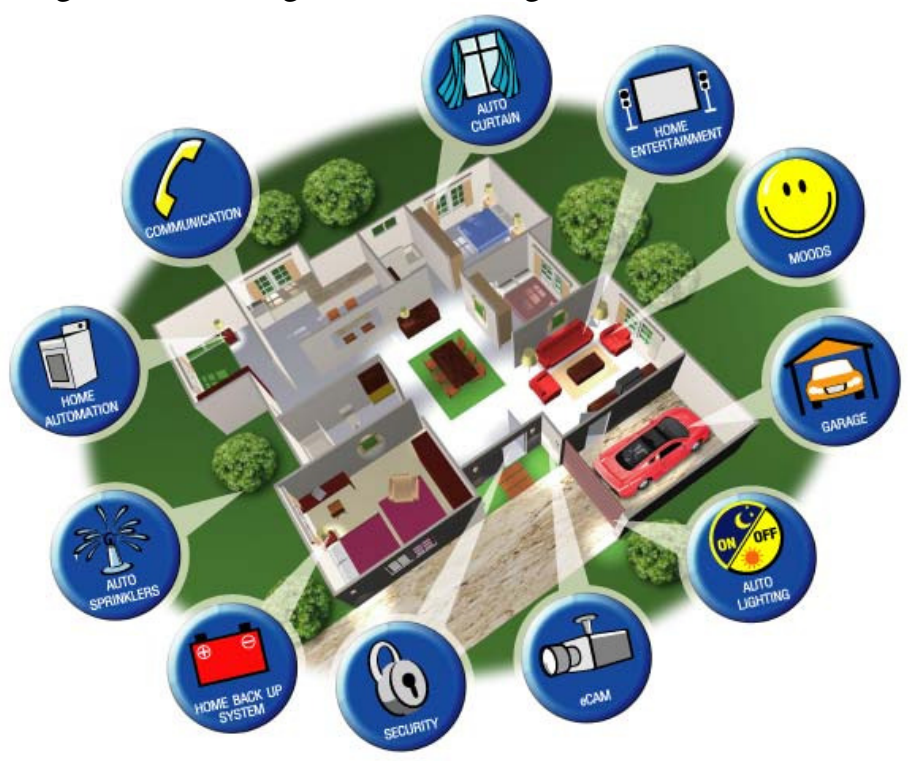

Figure 1: An example of home automation

\subsection{Highlights of 802.15.4 standard}

This standard is a specification of the PHYsical layer (PHY) and Medium Access Control (MAC) sub-layer for low data rate wireless connectivity among relatively simple devices that consume minimal power and typically operate in a Personal Operating Space (POS) of 10 meters or less. The network can be a one-hop star, or, when lines of communication exceed 10 meters, a self-configuring, multi-hop ad-hoc network.

\subsection{ZigBee Value Addition}

The ZigBee Alliance defines the network (NWK), security, and application profile layers for the 802.15.4-based system. Two routing (Tree and Mesh routing) protocols have been defined. The algorithms are kept lightweight as devices are expected to be simple and have small memory. 
International Journal of Wireless \& Mobile Networks (IJWMN) Vol. 5, No. 1, February 2013

Note that traditional table-driven ad hoc wireless routing protocols such as DSDV [2], CGSR, WRP, AODV [1, 3], require significant amount of memory for maintaining routing tables and hence are not suitable for these types of devices. Source-initiated routing such DSR [4], LMR, TORA, ABR, SSR may be considered as alternatives as they do not use any routing table. But, for a long network, incorporating routing information in the packet $y$ the source is not practical due to the limitation on maximum packet size (16 bytes). Moreover, data rate is very low (e.g. not more that 100 packets throughout the day for a personal area network for home appliance) that requires a relatively light-weight routing algorithm which incurs an overhead as small as possible.

\subsection{ZigBee Device Types}

A device in a Zigbee network can be physically a Full Function Device (FFD) or a Reduced Function Device (RFD). An FFD typically has more resources than an RFD. Logically a Zigbee-device can be a

-ZigBee coordinator: 802.15.4 PAN coordinator for a ZigBee network—must be an FFD.

-ZigBee router: 802.15.4 FFD that is not the ZigBee coordinator but capable of being so and participates in mesh routing.

-ZigBee end-device: 802.15.4 RFD or FFD that is not a ZigBee coordinator..

\subsection{Topologies Supported by Network Layer.}

The ZigBee network (NWK) layer supports star, tree and mesh topologies. In a star topology, the network is controlled by one single device called ZigBee coordinator. The ZigBee coordinator is responsible for initiating and maintaining network. All other devices, known as end devices, directly communicate with the ZigBee coordinator. In mesh and tree topologies, the ZigBee coordinator is responsible for starting the network and for choosing certain key network parameters but the network may be extended through the use of ZigBee routers. In tree networks, routers move data and control messages through the network using a hierarchical routing strategy. Mesh networks allow full peer- to-peer communication.

\subsection{Network Address Assignment}

Network addresses are assigned using a distributed addressing scheme that is designed to provide a finite sub-block of network addresses to every potential parent. These addresses are unique within a particular network and are given by a parent to its children. The ZigBee coordinator determines the maximum number of children (nwkMaxChildren) that any device within its network is allowed. Of these children, a maximum of nwkMaxRouters can be routercapable devices while the rest will be reserved for end devices. But no hint is given how these parameters are determined. Every device has an associated depth, which indicates the minimum number of hops to reach ZigBee coordinator. The ZigBee coordinator itself has a depth of zero, while its children have a depth of one. Multi-hop networks have a maximum depth that is greater than one. The ZigBee coordinator also determines the maximum depth (nwkMaxDepth) of the network.

Given the following network parameters:

$C_{m}=$ maximum number of children a ZigBee device may accept, $n w k M a x C h i l d r e n$

$L_{m}=$ maximum depth in the network, nwkMaxDepth 
International Journal of Wireless \& Mobile Networks (IJWMN) Vol. 5, No. 1, February 2013

$R_{m}=$ maximum number of router-capable-children a ZigBee device may accept, nwkMaxRouters

$E_{m}\left[=\left(C_{m}-R_{m}\right)\right]=$ maximum number of end-devices a ZigBee device may accept as children

We may compute the function, $C_{\text {skip }}(d)$, essentially the size of the address sub-block distributed by each parent at depth $d$ to each of its router-capable child devices, as follows:

$$
C_{s k i p}(d)= \begin{cases}0 & : d=L_{m} \\ 1+C_{m} .\left(L_{m}-d-1\right), & : R_{m}=1, d \neq L_{m} \\ \frac{1+C_{m}-R_{m}-C_{m} \cdot R_{m}^{L_{m}-d-1}}{1-R_{m}} & : R \neq 1, d \neq L_{m}\end{cases}
$$

If a device has a $C_{s k i p}(d)$ value of zero, then it shall not be capable of accepting children and shall be treated as a ZigBee end device. A device that has a $C_{s k i p}(d)$ value greater than zero may accept child devices and may assign addresses to them differently depending on whether the child device is router-capable or not. Network addresses are assigned to router-capable child devices using the value of $C_{\text {skip }}(d)$ as an offset.

A router-capable device having address $A_{\text {parent }}$ at depth $\mathbf{d}$ assigns addresses to its $\mathbf{n}^{\text {th }}$ child $\mathbf{A}_{\mathbf{n}}$ at depth $\mathbf{d}+\mathbf{1}$ in the following way:

END-DEVICE CHILD:

$$
A_{n}=A_{\text {parent }}+C_{\text {skip }}(d) R_{m}+n: 1 \leq n \leq E_{m}
$$

ROUTER-CAPABLE CHILD:

$$
A_{n}=A_{\text {parent }}+C_{\text {skip }}(d)(n-1)+1 \quad: 1 \leq n \leq R_{m}
$$

The first router-capable child gets address $A_{\text {parent }}+1$ and subsequent router-capable children get addresses separated by $C_{\text {skip }}(d)$. End-devices get addresses starting from $A_{\text {parent }}+1+C_{\text {skip }}(d) * R_{m}$ separated by 1 . Such example is shown in Figure. 3 and Figure 6.

\subsection{Tree Routing Mechanism}

For hierarchical routing, if the destination is a descendant of the device, the device shall route the frame to the appropriate child. Trivially, every other device is a descendant of the ZigBee Coordinator and no device is a descendant of any ZigBee end-device.

Refer to (1), (2) and (3), taking a routing decision is very simple. A target device with address $D$ is a descendant of a ZigBee router with address $A$ at depth $d$ if

$$
A<D<A+C_{\text {skip }}(d-1)
$$

This follows because the parent $P$ at depth $(d-1)$ of the device $X$ with address $A$, gives the address $A$ to $X$ and the address $A+C_{\text {skip }}(d-1)$ to the "closest" router-capable-sibling of $X$ that is 
also a child of $P$. The address sub-block $\left[A+1, A+C_{\text {skip }}(d-1)-1\right]$ is reserved for the descendants of $X$.

If the destination is a descendant of the receiving device, the address $N$ of the next hop device is determined as:

$$
N=\left\{\begin{array}{l}
D, \text { if } D>A+R_{m} \times C_{\text {skip }}(d) \text { for end devices } \\
A+1+\left\lfloor\frac{D-(A+1)}{C_{\text {skip }}(d)}\right\rfloor \times C_{\text {skip }}(d), \text { otherwise }
\end{array}\right.
$$

If equation (5) is not satisfied, next hop device is its parent. We look below at the derivation of formula (6):

The next-hop address is $N=A+1+k C_{\text {skip }}(d)$, i.e. the packet is to be routed to the router-capablechild with address $A+1+k C_{\text {skip }}(d)$ if $A+1+k C_{\text {skip }}(d) \leq D<A+1+(k+1) C_{\text {skip }}(d)$

This is because the device with address $A+1+k C_{\text {skip }}(d)$ gets address sub-block $\left[A+1+k C_{\text {skip }}(d)\right.$,

$$
\begin{aligned}
& A+1+(k+1) C_{\text {skip }}(d) \text {-1] i.e. } \quad N=A+1+k C_{\text {skip }}(d), \text { Where } k \leq \frac{D-A-1}{C_{\text {skip }}(d)}<k+1 \\
& \Rightarrow k=\left\lfloor\frac{D-A-1}{C_{\text {skip }}(d)}\right\rfloor
\end{aligned}
$$

So, next hop router can be determined by using an equation and the complexity of this scheme is constant. Moreover, there is no routing table and that way searching procedure is completely eliminated. In spite of this, tree routing has several limitations as described in the next section.

\subsection{Problem Definition}

The fundamental query that arises regarding tree routing is "how to choose the values of $C_{m}$ and $R_{m}$ ?". In many cases, before actually forming the network, we have very little or no idea about the following parameters:

- Number of end devices that will join to a router

- Number of routers that will join to a router

- Depth of the tree network

Also once the value is chosen the address distribution will be symmetric and it will not be able to support asymmetric structures required in mine field, glaciers sea bed, building premises etc.

Because an address sub-block cannot be shared between devices, it is possible that one parent exhausts its list of addresses while a second parent has addresses that go unused. A parent having no available addresses shall not permit a new device to join the network. In this situation, the new device shall find another parent. If no other parent is available within transmission range of the new device, the device shall be unable to join the network unless it is physically moved or there is some other change. 
This routing technique uses a fixed address assignment i.e. the no of child are fixed at any level and hence the address range, it is possible to have a network in which the no of child per parent increases as the depth increases, in this kind of network a large range of address will remain unused at the top level

Finally, due to the fact that the tree, it is not dynamically balanced, the possibility exists that certain installation scenarios, such as long lines of devices, may exhaust the address capacity of the network long before the real capacity is reached.

Following section shows a typical network formation where for a part of the tree number of node at the higher layer is relatively high but no nodes are present at the lower level.

Let $\mathrm{Cm}=2, \mathrm{Lm}=4, \mathrm{Rm}=2$

\begin{tabular}{|l|l|}
\hline Depth in the Network & Offset Value [Cskip(d)] \\
\hline 0 & 15 \\
\hline 1 & 7 \\
\hline 2 & 3 \\
\hline 3 & 1 \\
\hline 4 & 0 \\
\hline
\end{tabular}

Table 1 -Cskip

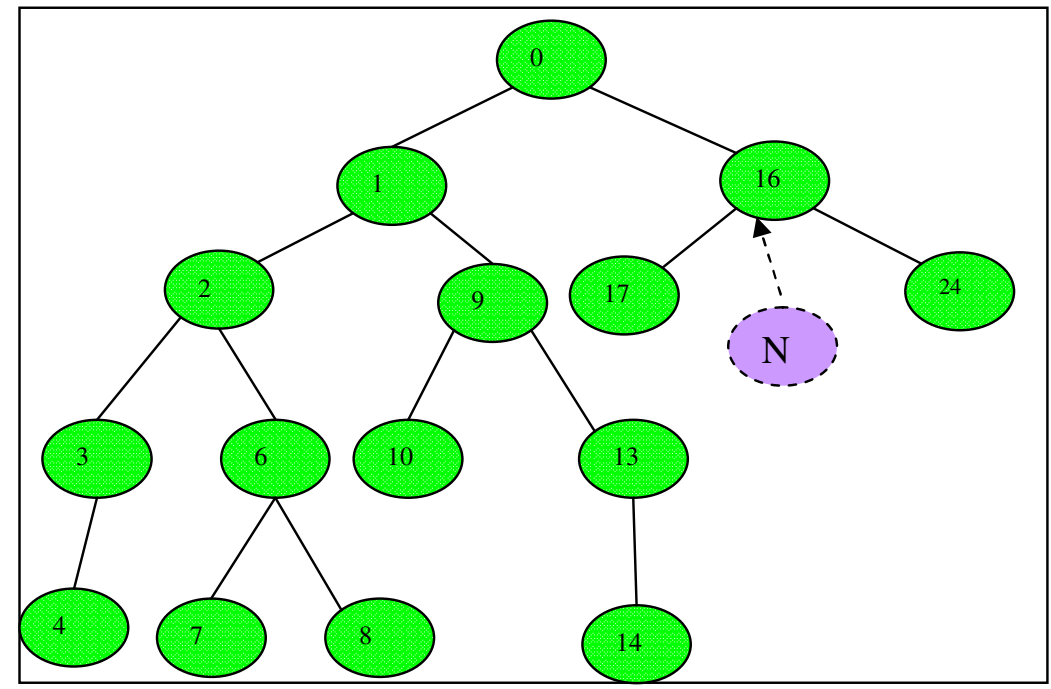

Figure 3: Limitation on maximum child for ZigBee tree networks

\section{Related works}

Wireless networks have rapidly gained popularity since their introduction in 1970s. However, an investigation into low-cost, low-rate, low-power PAN is relatively new. 
International Journal of Wireless \& Mobile Networks (IJWMN) Vol. 5, No. 1, February 2013

In [6], we have provided a unified address borrowing scheme which can be easily applied to grow the network beyond 16 hops and overcome the address exhaustion problem by borrowing address. A routing algorithm based on mobile IP, is also proposed.

In [7], we extended the Tree routing proposed by ZigBee for the networks to be harsh and asymmetric.

\section{Proposed Address Reorganization Algorithm}

In this paper we have extended our solution of single level address re-organization to multiple levels with out any extra over head such as routing table. The next hop address is calculated using mathematical formula only .This algorithm will allow the formation of any asymmetric network as per the need and thus remove the limitation of symmetric address distribution and formation. In real world most of the networks area is asymmetric for example in a building the lower floors can have more rooms compared to upper floors and part of ground floor may have canteen/gym etc so does not require any network or a paddy field can have some irregular shape, proposed address reorganization technique is capable of handling all these and can be applied to all such asymmetric networks.

In this scheme a node will be allowed to join a network at a node even if it has reached maximum no child by Address reorganization. This scheme can be used in part of the network where the network wants to grow in breadth rather than in depth i.e. we are expecting that the depth will be less than $\mathrm{Lm}$ at that part of the network. In Figure 3 the maximum length of the path from root which goes via Node 16 is two i.e. the depth of the network at that part is 2(less than Lm) which suggests that at that part of the network the growth is around the breadth and not in depth. We can apply our algorithm at that part.

In proposed address reorganization scheme any parent can increase its no of child device by reorganizing its address by any level so it is much more flexible.

\subsection{Overview of the Algorithm}

The Node which has reached its maximum child and wants to expand its breadth will use the next level Cskip value while distributing the address to its child i.e. if the node is at level $\mathrm{K}$ it will use the Cskip value for $\mathrm{K}+1$, its immediate child will use $\mathrm{K}+2$ and so on till $\mathrm{Lm}-1$.By doing this the Node has gained one level of address which it can use for adding new child.In Figure 3 if a new node wants to join the network at Node 16 then it will not be allowed as Node 16 has exhausted its address even though free address is available at other node such as Node 17 or Node 24.Our proposed algorithm could be used in such scenarios.

After address reorganizing the maximum no of child that can be added to that node will be $\mathrm{Rm} * \mathrm{Rm}+\mathrm{Cm}$

For example if node 16 goes for address reorganization as it is at level 1 its original Cskip value is 7 but it will use its next level Cskip value i.e. level 2 which is 3 , and the maximum no of child it can have will be $2 * 2+2=6$ and the network depth at that part will be Lm-1=3.

So after address reorganization node 16 will be able to add 4 more children. Following Figure 4 shows the structure of the network after address reorganization 


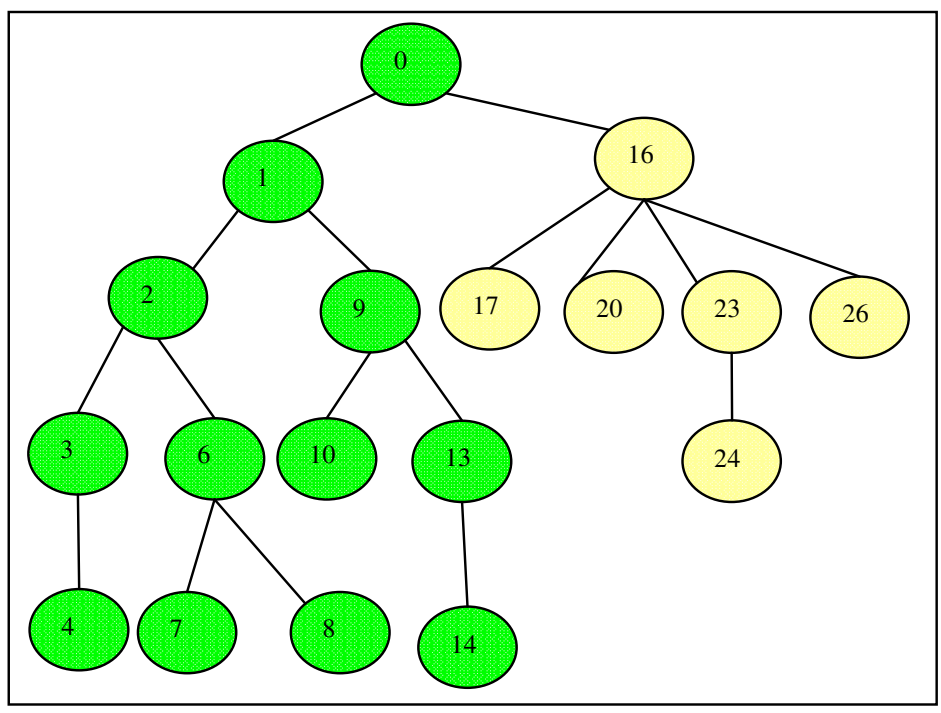

Figure 4 Address Reorganization

\subsection{Required Data Structure}

Transition table: This table will be maintained at the Node which has reorganized its address e.g. Node 16 in Figure 4. It will have 2 column Name and value.

\begin{tabular}{|l|l|}
\hline Name & Value \\
\hline Actual Cskip(Cskip) & 7 \\
\hline Pseudo Cskip(Pcskip) & 3 \\
\hline Actual Level(d) & 1 \\
\hline Pseudo Level(Pd) & 2 \\
\hline
\end{tabular}

Table 2 -Transition Table

This table will have 4 rows and will be used by the address reorganizing Node while assigning address to its child devices.

In the above table Pseudo level $(\mathrm{Pd})=\mathrm{d}+1$ and $P_{\text {cskip }}(d)=C_{\text {skip }}(d+1)$

All the child node of this will be at pseudo level Pd+1and there child at level Pd+2 and so on. That is the node which has re organized its address will physically be at level d but will virtually consider it to be at level $\mathrm{d}+1$.

\subsection{Address Distribution}

In this variant if the node has reorganized its address by level ' $v$ ' the maximum no of child that the node can have is $\left(R_{m}^{v+1}+R_{m}\right)$

At any Node if it has not reorganized its address or any of its parents have not reorganized its address then the address assignment will follow equation 2 and 3 for Router and end device respectively. 
If the Node has reorganized its address by level ' $v$ ' then the address assigned to its router capable device will be as follows:

$$
\begin{aligned}
& \text { For } R^{\text {th }} \text { routing capable child if } \mathrm{R} \text { is }<=R_{m}^{v+1}+1 \\
& A_{R^{\text {th }}}=A_{\text {parent }}+P_{\text {cskip }}(d) \cdot\left(R^{\text {th }}-1\right)+1 \\
& \text { Else if } R_{m}^{v+1}+1<R<=R_{m}^{v+1}+R_{m} \\
& \text { Then address is given by } A_{R^{\text {th }}}=A_{\text {parent }}+P_{\text {cskip }}(d) R_{m}^{v+1}+1+E \\
& \text { Where } \mathbf{G}=\left(R_{m}+R_{m}^{2}+\ldots \ldots \ldots R_{m}^{v}\right) \& \text { for } v>3 \\
& E=\left(C_{m}-R_{m}+1\right) \cdot\left(1+R_{m}+R_{m}^{2}+\ldots . R_{m}^{v-1}\right) \cdot\left(R-R_{m}^{v+1}-1\right) \\
& \text { for } v=1, \quad E=\left(C_{m}-R_{m}+1\right) \cdot\left(R-R_{m}^{v+1}-1\right) \\
& \text { for } v=2, \quad E=\left(C_{m}-R_{m}+1\right) \cdot\left(1+R_{m}\right) \cdot\left(R-R_{m}^{v+1}-1\right) \\
& \text { for } v=3, \quad E=\left(C_{m}-R_{m}+1\right) \cdot\left(1+R_{m}+R_{m}^{2}\right) \cdot\left(R-R_{m}^{v+1}-1\right)
\end{aligned}
$$

These nodes will mark their relative position as 1 . i.e. they are at 1 level below the node which has reorganized its address. The child node of this node will mark its relative position as two and so on. For example in Figure-7 Node 42 and 51 are at relative level 1, node 43, 46, 52 and 55 are at relative level two. The Cskip value for a node at relative level 'e' for' $v$ ' $>3$ is given by

$$
\begin{array}{ll}
R_{\text {skip }}(e)=\left(C_{m}-R_{m}+1\right) .\left(1+R_{m}+R_{m}^{2}+\ldots \ldots \text { upto }(v-e) \text { term }\right) \\
\text { for } v \text { - }=1, & R_{\text {skip }}(1)=\left(C_{m}-R_{m}+1\right) . \\
\text { for } v \text {-e }=2, & R_{\text {skip }}(1)=\left(C_{m}-R_{m}+1\right) .\left(1+R_{m}\right) \\
\text { for } v \text {-e }=3, & R_{\text {skip }}(1)=\left(C_{m}-R_{m}+1\right) .\left(1+R_{m}+R_{m}^{2}\right)
\end{array}
$$

Network address to the end device is given in a sequential manner and the address given to nth end device is given by the following equation.

$$
\begin{aligned}
A_{n} & =A_{\text {Parent }}+P_{\text {cskip }}(d) \cdot R_{m}^{v+1}+n+F \\
1 & \leq n \leq C m-R m \text { And } A_{\text {parent }} \text { is address of parent And } F=\left(C_{m}-R_{m}+1\right) \cdot G
\end{aligned}
$$

All the nodes which are descendent of first $R_{m}^{v+1}$ child of that reorganizing node will use the subsequent depth and Cskip values, and the node which are descendent of next Rm child will use Rskip value for address distribution. Please refer to Figure-7 for network formation. In that node 1 has reorganized its address by level 2 so the no of router capable device that can be attached to it is $R_{m}^{3}+R_{m}=10$ among which 8 node have Pseudo Cskip here $P_{c s k i p}=5$ and the other 2 child Node will have Relative Cskip here the value is $R_{\text {skip }}(1)=3 \times 3=9$ according to Equation 17 i.e. node $\&$ which is among $1^{\text {st }} 8$ child node will use $P_{c s k i p}=5$ and node 42 will use $R_{\text {skip }}(1)=3 \times 3=9$ for address distribution.

\subsection{Additional Data Structure}

In this type one additional register $R_{p o s}$ will be required for storing the relative position. This register's value will be set only if the Rth Node is a child of the address reorganizing node and if $R_{m}^{v+1}<R<=R_{m}^{v+1}+R_{m}$ or child of a node which satisfies the above two conditions for example in Figure-7 node 42 has $R_{\text {pos }}=1$ Node 43 has $R_{\text {pos }}=2$ 


\subsection{Routing in Reorganized Network}

At any Node if it has not reorganized its address then the routing will follow the normal process i.e. it will follow equation 4,5and 6 .

If the Node has reorganized its address then routing will be as follows:

If the destination is a descendant of the device, the device shall route the frame to the appropriate child. If the destination is not a descendant, the device shall route the frame to its parent. For a ZigBee router with address A at depth d, and pseudo depth Pd if the following logical expression is true, then a destination device with address $\mathrm{D}$ is a descendant:

$$
A<D<A+C_{\text {skip }}(d-1)
$$

If it is determined that the destination is a descendant of the receiving device then it is checked if the destination is a child end device using the formula

$D>A+R_{m}^{v+1} \cdot P_{\text {cskip }}\left(P_{d}\right)+G .\left(C_{m}-R_{m}+1\right)$,

If it is a child end device then the address $\mathrm{N}$ of the next hop device is given by: $\mathrm{N}=\mathrm{D}$.

Otherwise,

If the destination address $A<D<=A+P_{c s k i p}\left(P_{d}\right) \cdot R_{m}^{v+1}$

then the next hop address is given by:

$N=A+1+\left\lfloor\frac{D-(A+1)}{P_{c s k i p}\left(P_{d}\right)}\right\rfloor \times P_{c s k i p}\left(P_{d}\right)$

else the next hop address is given by

$N=Z+\left\lfloor\frac{D-Z}{R_{c s i k}}\right\rfloor \times\left(R_{c s k i p}\right)$

Where $Z=A+1+R_{m}^{v+1} \times P_{\text {cskip }}\left(P_{d}\right)$

All the nodes which are descendent of first $R_{m}^{v+1}$ child of that reorganizing node shall use there pseudo depth and the node which are descendent of next $\mathrm{Rm}$ child shall use relative depth and relative Cskip value in place of Cskip in equation 4,5,6,7 for routing purpose, no other changes are needed .

A node will use $R_{\text {pos }}$ register to decide if it needs to use $P_{\text {cskip }}$ or $R_{\text {skip }}(e)$ for routing. If the $R_{p o s}$ register contains a not null value then it will use $R_{\text {skip }}(e)$ else it will use $P_{c s k i p}$ if it is a descendent of address reorganized node and $C_{\text {skip }}$ otherwise.

For example in Figure-7 say node 71 wants to send some data to Node 34 it will go via the following path. Figure $\mathbf{- 5}$ shows the flow chart for the routing.

$71 \Rightarrow 70 \Rightarrow 64 \Rightarrow 63 \Rightarrow 62 \Rightarrow 0 \Rightarrow 1 \Rightarrow 32 \Rightarrow 34$.In Figure 8 the message flowing path has been highlighted in dark violet.

At Node 1 routing decision will be taken depending on equation $(\mathbf{2 0 , 2 1 , 2 2 , 2 3 , 2 4 )}$ at all the other nodes routing will follow equation 3,4,5.

Again If Node 14 wants to send some data to node 114 it will

follow the following path.

$14 \Rightarrow 12 \Rightarrow 1 \Rightarrow 0 \Rightarrow 62 \Rightarrow 92 \Rightarrow 106 \Rightarrow 112 \Rightarrow 114$ In Figure 8 the message flowing path has been highlighted in dark blue. 
International Journal of Wireless \& Mobile Networks (IJWMN) Vol. 5, No. 1, February 2013

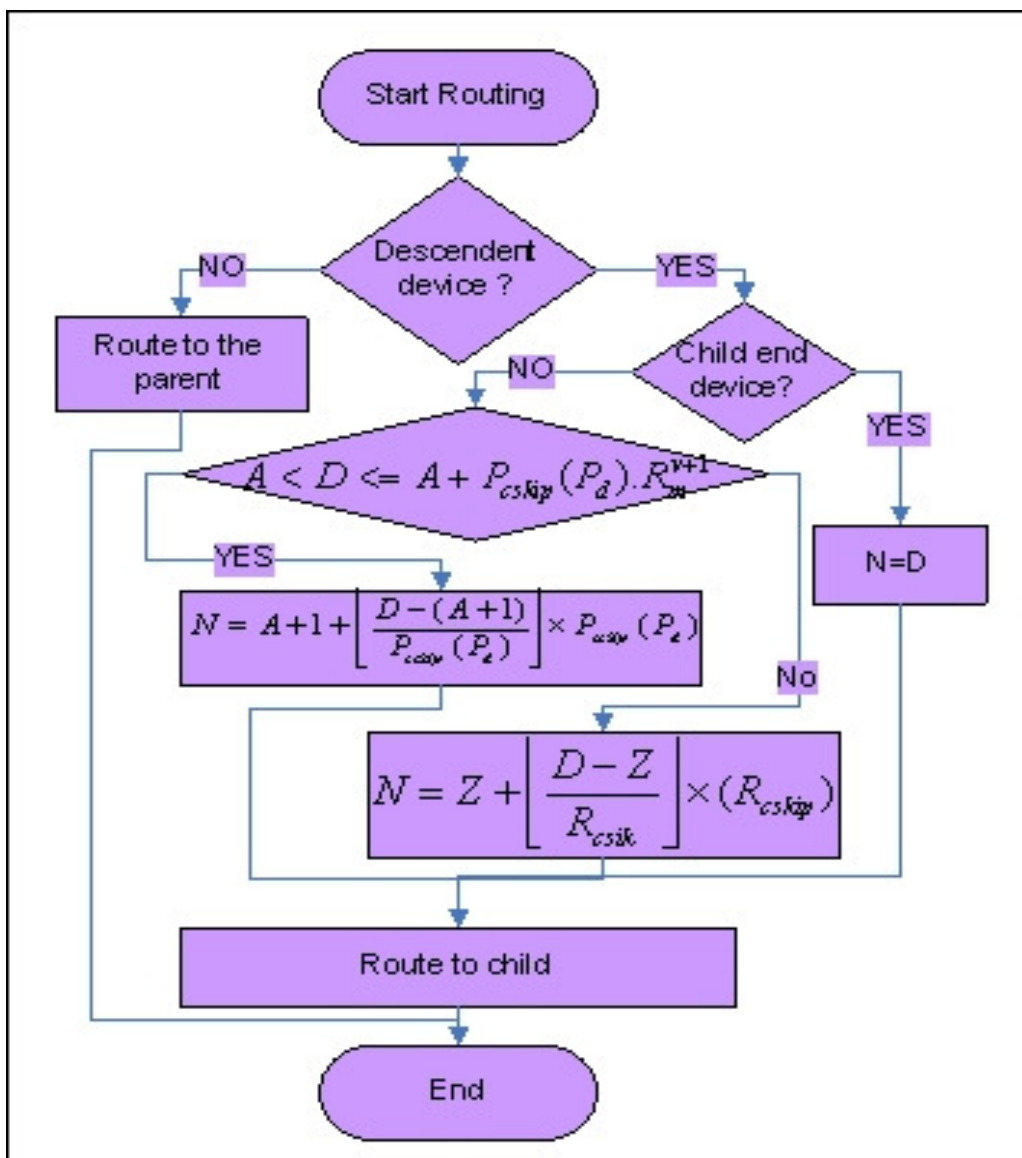

Figure 5: Routing in address Reorganized Network

\begin{tabular}{|l|l|l|l|l|l|}
\hline$C_{\text {skip }}(0)$ & $C_{\text {skip }}(1)$ & $C_{\text {skip }}(2)$ & $C_{\text {skip }}(3)$ & $C_{\text {skip }}(4)$ & $C_{\text {skip }}(5)$ \\
\hline 61 & 29 & 13 & 5 & 1 & 0 \\
\hline
\end{tabular}

Table 3: Cskip Value at various levels 


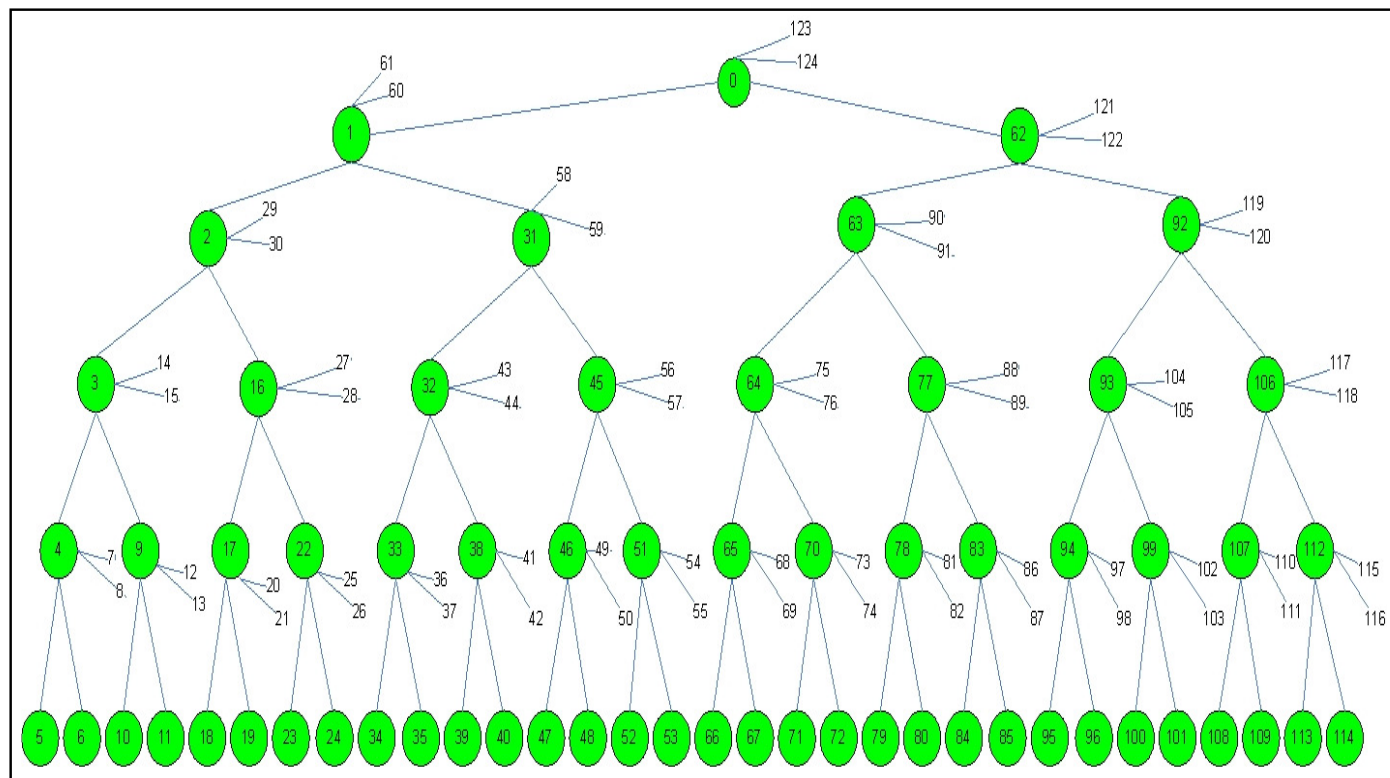

Figure 6: Address Distribution without address Reorganization

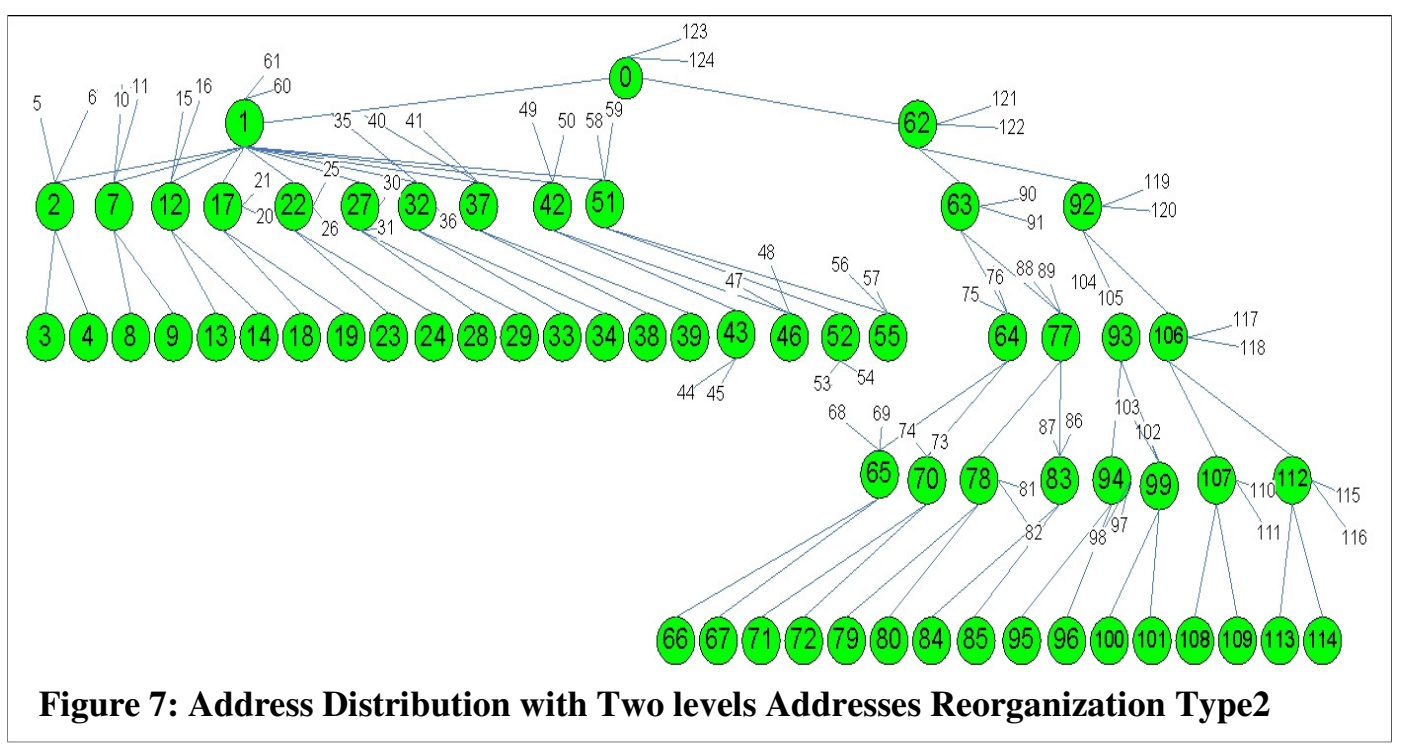




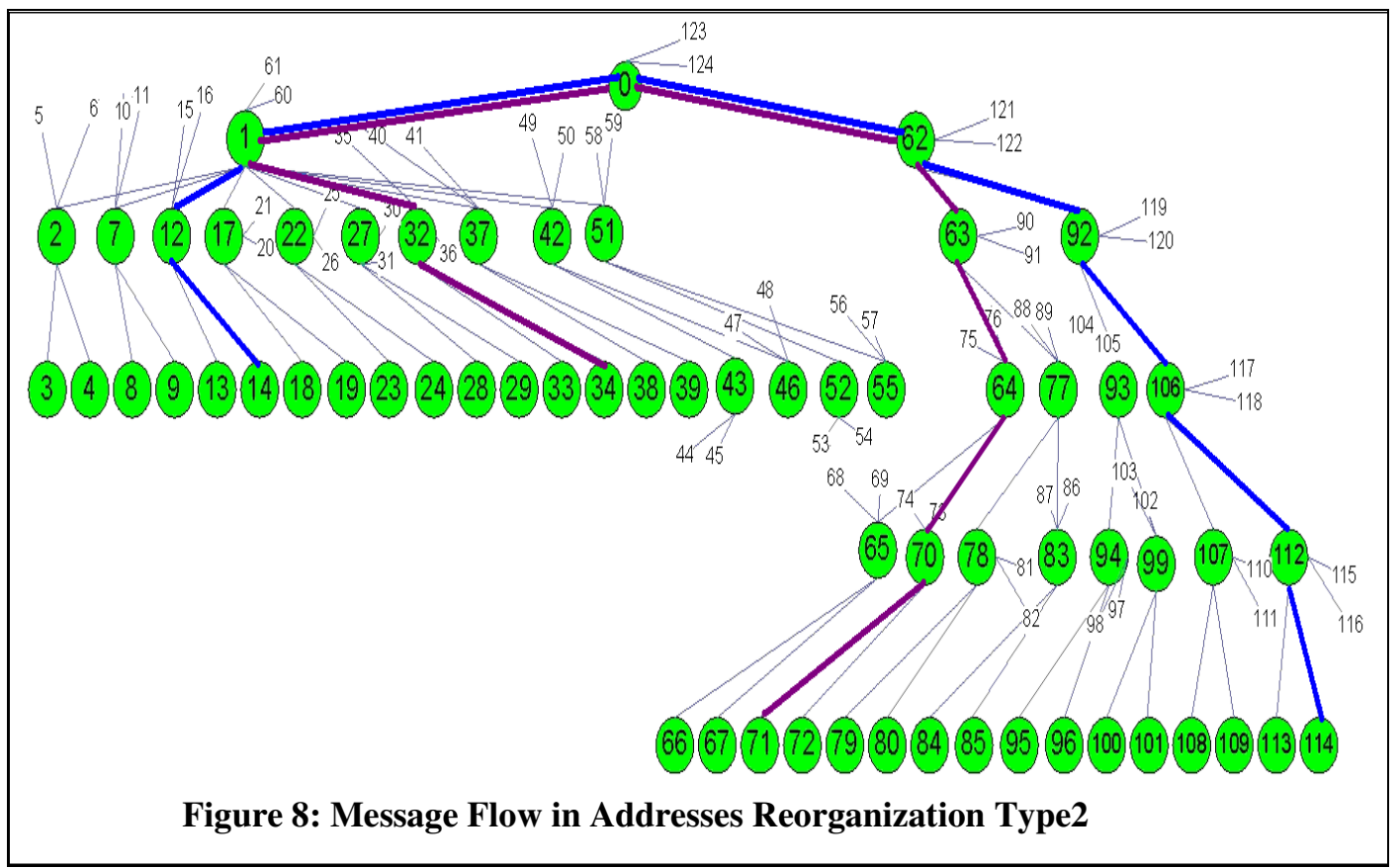

\subsection{Analysis of Overhead}

In this address re-organized network if the node has reorganized its address by level ' $\mathrm{v}$ ' the maximum no of router capable child that the node can have is $\left(R_{m}^{v+1}+R_{m}\right)$. For example in normal tree network (Figure 6 ) the Node 1 can have at most 2 router capable device attached to it. But if the requirement is to add more router capable devices at this node then it can reorganize its address by any level .In Figure 7 we have shown a re-organized network where Node 1 has re-organized its address with 2 levels i.e. $\mathrm{v}=2$.and $\mathrm{Rm}=2$. So it can now have $\left(2^{2+1}+2\right)=10$ router capable device. Following table shows the relation ship between different address re-organization level(v) and the max no of router capable child that a reorganized node could have for a given $\mathrm{Rm}$.For example if for a network $\mathrm{Rm}$ is chosen as 2.then If any node wishes to re-organize its address by level 1 it can now have 6 router capable child. If it has re-organized its address by level 3 then it can have 18 router capable child and so on. 
International Journal of Wireless \& Mobile Networks (IJWMN) Vol. 5, No. 1, February 2013

\begin{tabular}{|l|l|l|}
\hline $\begin{array}{l}\text { No of re } \\
\text { organization } \\
\text { Level (v) }\end{array}$ & $\begin{array}{l}\text { Max no of } \\
\text { Router } \\
\text { capable Child } \\
\text { without } \\
\text { address } \\
\text { reorganization }\end{array}$ & $\begin{array}{l}\text { Max No of Router capable } \\
\text { Child with address } \\
\text { reorganization }\end{array}$ \\
\hline 1 & 2 & 6 \\
\hline 2 & 2 & 10 \\
\hline 3 & 2 & 18 \\
\hline 4 & 2 & 34 \\
\hline 1 & 3 & 12 \\
\hline 2 & 3 & 30 \\
\hline 3 & 3 & 84 \\
\hline 4 & 3 & 246 \\
\hline
\end{tabular}

Table 4 : Max Router capable device.

No of Router capable device at any level $\mathrm{v}$ is $R_{m}^{v}$ this entire node could re-organize its address by 1 to $\mathrm{Lm}-\mathrm{v}-1$ level so there will be (Lm-v-1) $R_{m}^{v}$ pattern that could be formed at level v. So if we consider each router capable devices of all $\mathrm{Lm}$ levels then all together the no of patterns that could be formed will be.

$$
\sum_{v=1}^{L_{m}-2} R_{m}^{v}\left(L_{m}-v-1\right)
$$

Following table shows the relation ship between the no of level and the no of different pattern that could be supported for a given Rm, Lm. For example if $\mathrm{Rm}=2, \mathrm{Lm}=3$ then the different pattern formation additional to the normal network is 2 .

\begin{tabular}{|l|r|}
\hline Network Parameter & $\begin{array}{l}\text { No of Different } \\
\text { Structure Possible } \\
\text { with address re- } \\
\text { organization }\end{array}$ \\
\hline$R M=2, L M=3$ & 2 \\
\hline$R M=2, L M=4$ & 8 \\
\hline$R M=2, L M=5$ & 22 \\
\hline$R M=2, L M=6$ & 52 \\
\hline$R M=2, L M=7$ & 114 \\
\hline$R M=2, L M=8$ & 240 \\
\hline$R M=2, L M=9$ & 494 \\
\hline$R M=2, L M=10$ & 1004 \\
\hline$R M=2, L M=11$ & 2026 \\
\hline$R M=2, L M=12$ & 4072 \\
\hline$R M=2, L M=13$ & 8166 \\
\hline$R M=2, L M=14$ & 16356 \\
\hline$R M=2, L M=15$ & 32738 \\
\hline$R M=2, L M=16$ & 85984 \\
\hline
\end{tabular}

Table 5: Different Network Formation 
International Journal of Wireless \& Mobile Networks (IJWMN) Vol. 5, No. 1, February 2013

Figure 9 : No of Different Structure Possible with address re-organization

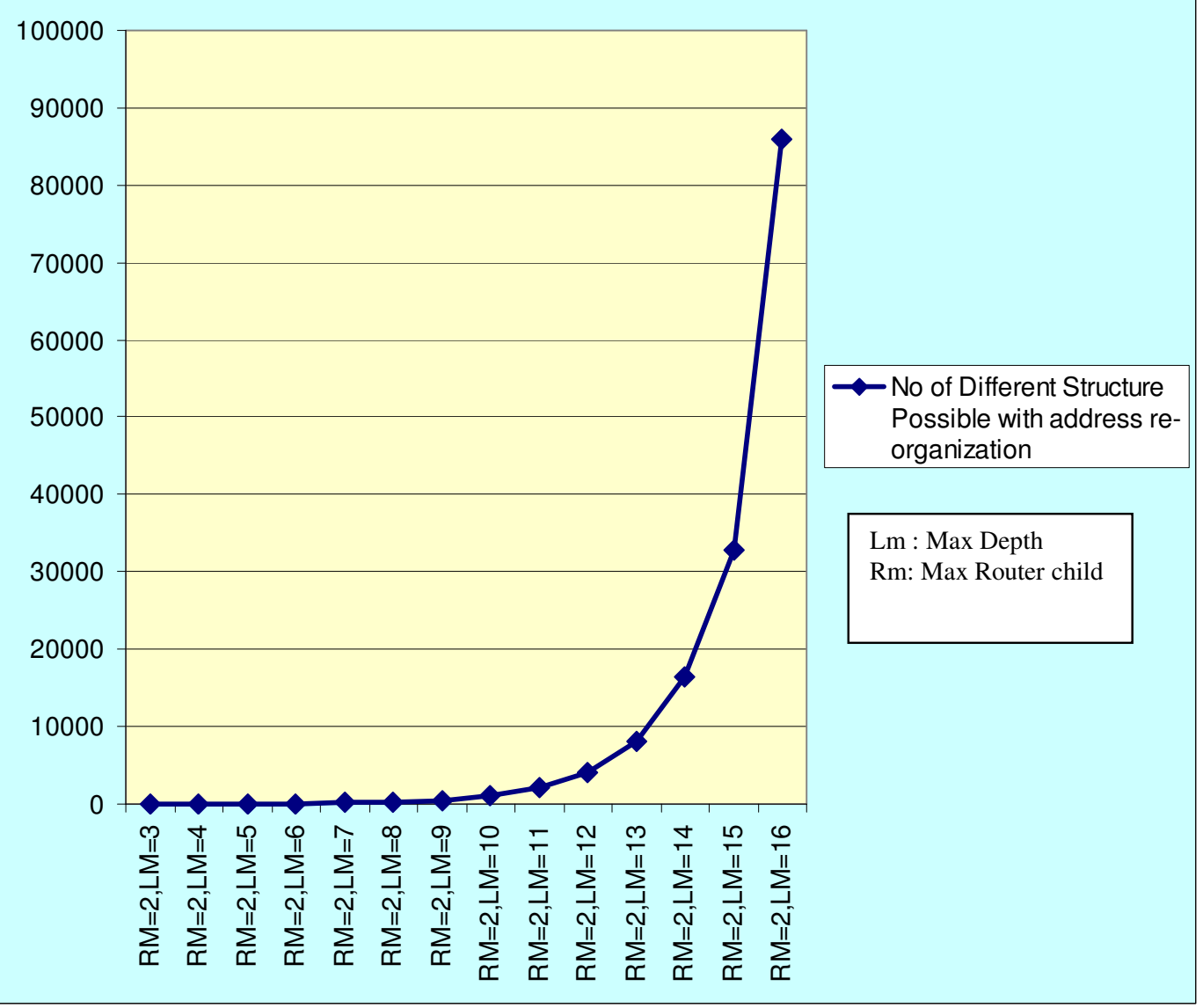

\section{CONCLUSIONS}

Sensornets are closely coupled to the physical world and can directly impact our Personal Operating Space (POS). One of the major advantages of IEEE802.15.4 is that it uses free 2.4 GHz ISM band at the Physical layer. Nodes in a sensornet are low cost and the performance of this architecture is already well-proven.

The wireless network with minimum data rate, reduced energy consumption and minimum cost is formed by IEEE 802.15.4 standard. The distinctive characteristics of the standard makes it more approving module for wireless sensor networks and remote monitoring applications. It offers a minimum power, economic and a consistent protocol for wireless connectivity among low-cost, permanent and moveable devices. These devices can figure out into a sensor network or wireless personal area network (WPAN). After a great success in personal operating space (POS), researchers are trying to use it in relatively broad areas such as industrial automation, tracking and monitoring systems, public telecom services etc. This is only possible if a large network can be formed. For this purpose it needs a suitable routing algorithm.

In this paper we have tried to mitigate the problem of symmetric structure and provided a unique technique of address reorganization which will help the network in a variety of different application scenarios staring from mine field to Glacier war field to building automation. The main advantage of this algorithm is that we can form many different network with the 
International Journal of Wireless \& Mobile Networks (IJWMN) Vol. 5, No. 1, February 2013

introduction of just two variable called Pseudo Cskip(Pcskip) and Pseudo Level(Pd) no other extra overhead such as routing table will not be required.

\section{ACKNOWLEDGEMENTS}

The authors would like to thank everyone, just everyone!

\section{REFERENCES}

[1] C. E. Perkins, E. Belding-Royer, and S. R. Das, "Ad hoc On-Demand Distance Vector (AODV) Routing”, http://www.ietf.org/rfc/rfc3561.txt, July 2003. RFC 3561.[AODV_1]

[2] C. E. Perkins and P. Bhagwat, "Highly Dynamic Destination-Sequenced Distance-Vector Routing (DSDV) for Mobile Computers”, Proceedings of ACM SIGCOMM, 1994.[C_E_DSDV]

[3] C. E. Perkins and Elizabeth Royer, "Ad-hoc On-Demand Distance Vector Routing”, Proceedings of the 2nd IEEE Workshop on Mobile Computing Systems and Applications, New Orleans, LA, February 1999. [AODV_2]

[4] D. B. Johnson, D. B. Maltz, "Dynamic Source Routing in Ad-hoc Wireless Networks”, Mobile Computing, T. Imielinski, H. Korth, Eds. Kluwer Academic Publishers, 1996, ch. 5, pp. 153181.[Johnson_DSR]

[5] D. Ganeshan, B. Krishnamachari, "Complex Behavior at Scale: An Experimental Study of LowPower Wireless Sensor Networks”, UCLA/CSD-TR 02-0013, UCLA Computer Science, 2002.[Low_Power]

[6] Debabrato Giri, Uttam Kumar Roy, "Address Borrowing In Wireless Personal Area Network", Proc. of IEEE International Anvanced Computing Conference, (IACC '09, March 6-7), Patiala, India, page no 1074-1079[ukr_borrowing]

[7] Debabrato Giri, Uttam Kumar Roy, "Single Level Address Reorganization In Wireless Personal Area Network", 4th International Conference on Computers \& Devices for Communication (CODEC-09), December 14-16, 2009, Calcutta University, India. [ukr_single]

[8] Ed Callaway, Paul Gorday, Lance Hester, Jose A. Gutierrez, Marco Naeve, Bob Heile and Venkat Bahl. "Home Networking with IEEE 802.15.4: A Developing Standard for Low-Rate Wireless Personal Area Networks”,IEEE Communications Magazine August 2002.[Low_Rate]

[9] Elizabeth Royer and C-K Toh, "A Review of Current Routing Protocols for Ad-Hoc Mobile Wireless Networks”, IEEE Personal Communications Magazine, April 1999.[Routing_Protocols]

[10] Gang Lu, Bhaskar Krishnamachari, Cauligi S. Raghavendra, "Performance Evaluation of the IEEE 802.15.4 MAC for Low-Rate Low-Power Wireless Networks", IEEE International Conference on Performance, Computing, and Communications, 2004.[Performace]

[11] IEEE 802.15.11 Standard: "Wireless Local Area Networks, 1999”. [WLAN]

[12] IEEE 802.15.1, Wireless Medium Access Control (MAC) and Physical layer (PHY) specifications for Wireless Personal Area Networks (WPANs) [Bluetooth]

[13] IEEE 802.15.4, Wireless Medium Access Control (MAC) and Physical Layer (PHY) Specifications for Low-Rate Wireless Personal Area Networks (WPANs) [WPAN]

[14] J. Heidemann, W. Ye and D. Estrin.”An Energy-Efficient MAC Protocol for Wireless Sensor Networks", Proceedings of the 21st International Conference of the IEEE Computer and Communications Societies (INFOCOM 2002), New York, NY, June 2002.[Energy]

[15] Jianliang Zheng and Myung J. Lee. "A Comprehensive Performance Study of IEEE 802.15.4", http://www-ee.ccny.edu/zheng/pub, 2004. [Comprehensive]

[16] 14, C. Schurgers, S. Park and M. B. Srivastava, "Energy-Aware Wireless Microsensor Networks", IEEE Signal Processing Magazine, Volume: 19, Issue: 2, March 2002.[Aware] 
International Journal of Wireless \& Mobile Networks (IJWMN) Vol. 5, No. 1, February 2013

[17] Shree Murthy, J. J. Garcia-Luna-Aceves, "An Efficient Routing Protocol for Wireless Networks", Mobile Networks and Applications, 1996.[Efficient]

[18] Uttam Kumar Roy, Debarshi Kumar Sanyal, Sudeepta Ray, "Analysis and Optimization of Routing Protocols in IEEE802.15.4" (Asian International Mobile Computing Conference (AMOC 2006), Jadavpur University, Kolkata, India [UKR_AMOC]

[19] ZigBee Alliance (ZigBee Document 053474r17) Draft Version 0.90: Network Specification, Jan 2008.[ZigBee]

[20] ZigBee Alliance (ZigBee Document 075307r07) Version 1.0: Telecom Applications Profile Specification, April 2010.[ZigBee Telecom]

[21] ZigBee Alliance (ZigBee Document 053516r12) Version 1.0: ZigBee Building Automation Application Profile, May 2011.[ZigBee Building Automation]

\section{Authors : Debabrato Giri}

Research Scholar,

Department of Information

Technology,

Jadavpur University

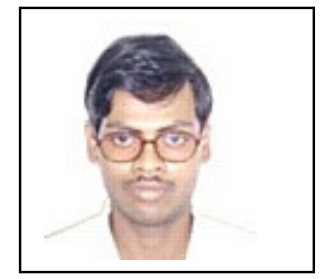

\section{Authors : Uttam Kumar Roy}

Currently working as an assistant professor in the Dept. of information Technology. Jadavpur University, Kolkata, India. Has completed his Ph. D in engineering from the same university and has nearly 10 years of teaching experience. He is the sole author of the book entitled "Web Technologies" published by Oxford University press in 2010. Contributed numerous research papers to various journals.

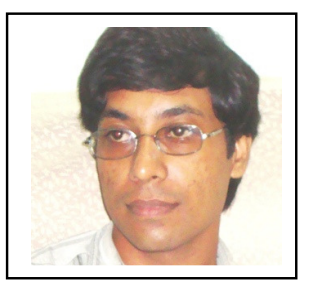

